\title{
Tratamento da Eclâmpsia: Estudo Comparativo entre 0 Sulfato de Magnésio e a Fenitoina
}

Treatment of Eclampsia: Comparative Study on the Use of Magnesium Sulfate and Phenytoin

João de Deus Valadares Netoํ․ Anna Maria Bertini²,

Wladimir Corrêa Taborda ${ }^{2}$, Joaquim Vaz Parente ${ }^{1}$

\section{RESUMO}

Objetivos: comparar a eficácia do sulfato de magnésio e da fenitoína no controle das convulsões em pacientes com eclâmpsia e avaliar os efeitos de sulfato de magnésio e da fenitoina sobre o prognóstico materno e perinatal em pacientes com eclâmpsia.

Métodos: estudo prospectivo, randômico e controlado no qual foram analisados, de forma comparativa, os resultados obtidos no tratamento anticonvulsivante da eclâmpsia em 77 mulheres tratadas com sulfato de magnésio ou fenitoina. As drogas que constituiram os dois esquemas terapêuticos foram distribuidas, na proporção de um para um, em caixas numeradas aleatoriamente que apresentavam caracteristicas semelhantes. À medida que cada paciente era admitida, uma caixa era aberta e o esquema nela contido administrado à paciente.

Resultados: observou-se que, no grupo tratado com sulfato de magnésio, 19,5\% das pacientes apresentaram recidiva de convulsões, ao passo que no grupo que usou fenitoina, 36,1\% manifestaram novas crises ( $p<0,05)$. As pacientes que foram tratadas com sulfato de magnésio apresentaram uma maior prevalência de hemorragia pós-parto $(14,7 \%)$ que as que utilizaram fenitoína $(2,7 \%)(p<0,05)$. Em relação aos recém-nascidos, $17,0 \%$ do grupo de mães tratadas com sulfato de magnésio apresentaram desconforto respiratório, contra 11,8\% no grupo que foi tratado com fenitoina ( $p>0,05)$.

Conclusões: o sulfato de magnésio mostrou-se mais eficaz que a fenitoina no controle e prevenção da recidiva de convulsões em pacientes com eclâmpsia, embora sua utilização esteja associada a maior prevalência materna de hemorragia pós-parto e desconforto respiratório neonatal. A fenitoina apresenta-se como droga alternativa para o tratamento de eclâmpsia nos casos em que houver contra-indicação ao uso do sulfato de magnésio.

PALAVRAS-CHAVE: Hipertensão arterial. Fenitoína. Eclâmpsia. Complicações do parto. Puerpério. Desconforto respiratório. Sulfato de magnésio.

\section{Introdução}

A doença hipertensiva específica da gravidez (DHEG) é uma entidade clínica própria da gestação humana que surge em geral na segunda metade do ciclo gestacional e acomete princi-

${ }^{1}$ Disciplina de Obstetrícia da Universidade Federal do Piauí Maternidade Dona "Evangelina Rosa"

${ }^{2}$ Disciplina de Obstetrícia da Universidade Federal de São Paulo

- Escola Paulista de Medicina

Correspondência:

João de Deus Valadares Neto

Rua Deusa Rocha, 1855 - Ilhotas

64014-180 - Teresina - PI palmente primigestas. Caracteriza-se pelo aparecimento da tríade hipertensão, edema e proteinúria (pré-eclâmpsia), podendo ter desfecho em convulsão e/ou coma (eclâmpsia).

De um modo geral a incidência de DHEG varia entre 5 e $10 \%{ }^{1,2}$. No caso particular da eclâmpsia, os estudos têm demonstrado uma ocorrência variando entre 1 para 100 e 1 para 1.700 gestações em países subdesenvolvidos ${ }^{3,4}$, ao passo que na Europa e em outras regiões desenvolvidas a eclâmpsia complica 1 a cada 2.000 gestações ${ }^{5}$.

O estudo da DHEG reveste-se de grande 
importância, tendo em vista a alta incidência e os elevados indices de mortalidade materna a ela associados, sobretudo em casos de eclâmpsia $^{6,7}$. No Reino Unido, a eclâmpsia é responsável por $10 \%$ das mortes maternas diretas e em todo o mundo ocorrem cerca de 50.000 mortes maternas a cada ano, em conseqüência de eclâmpsia. Por esta razão o manuseio adequado de mulheres com eclâmpsia, sobretudo no que diz respeito ao uso de anticonvulsivante, ocupa lugar de destaque ${ }^{8,9}$.

O tratamento anticonvulsivante da eclâmpsia teve início no começo do século XX, tendo sido o sulfato de magnésio a primeira droga utilizada com esta finalidade ${ }^{10}$. Ao longo do tempo vários esquemas foram propostos com esse objetivo, não havendo, contudo, consenso acerca de qual a melhor droga para o tratamento das convulsões eclâmpticas.

Na década de 1980, a fenitoína, um anticonvulsivante amplamente usado no controle da epilepsia, que não tem efeito sedativo, foi proposta para o manuseio de pacientes com eclâmp$\operatorname{sia}^{11}$.

Em 1990, apareceu o primeiro estudo comparativo do efeito anticonvulsivante do sulfato de magnésio e da fenitoína. Nele, quatro de onze pacientes com eclâmpsia tratadas com fenitoína apresentaram recidiva de crise convulsiva, ao passo que de onze pacientes tratadas com sulfato de magnésio nenhuma teve recorrência das convulsões ${ }^{12}$.

Em 1995, um grande estudo multicêntrico internacional comparou a eficácia do sulfato de magnésio em relação ao diazepam e à fenitoína no tratamento de pacientes com eclâmpsia. Neste estudo, 388 mulheres foram tratadas com sulfato de magnésio e 387 com fenitoína. No grupo que usou sulfato de magnésio, 22 mulheres apresentaram recidiva das convulsões, ao passo que no grupo que usou fenitoina, a recidiva das crises ocorreu em 66 mulheres $^{9}$.

Embora pareça claro, a partir destas observações, que o sulfato de magnésio é mais eficaz no tratamento da eclâmpsia que a fenitoína, torna-se necessária a realização de novas pesquisas, tendo em vista a necessidade de determinação efetiva da melhor droga para o tratamento das convulsões eclâmpticas.

O presente estudo tem por objetivo avaliar, de forma prospectiva, a eficácia do sulfato de magnésio e da fenitoína no controle e prevenção de recidivas de convulsões em pacientes com eclâmpsia, bem como o efeito destas substâncias sobre o prognóstico materno imediato e resultados perinatais.

\section{Pacientes e Métodos}

O presente trabalho consistiu de um estudo prospectivo, randomizado e controlado no qual foram analisados, de forma comparativa, os resultados obtidos no tratamento anticonvulsivante da eclâmpsia, em mulheres tratadas com sulfato de magnésio ou fenitoína.

Foram incluídas no estudo 77 pacientes portadoras de eclâmpsia, admitidas na Maternidade Dona "Evangelina Rosa", em Teresina, PI (MDER), no período compreendido entre março de 1996 e junho de 1998. Neste período foram internadas 126 pacientes com eclâmpsia, tendo sido excluídas do estudo aquelas que haviam sido tratadas antes da chegada à maternidade ou que receberam tratamento com drogas que não empregadas no protocolo de estudo. Todas as pacientes incluídas no estudo receberam um acompanhamento clínico até o momento da alta hospitalar.

Antes da realização da pesquisa, o projeto foi submetido à apreciação pela Comissão de Ética em Pesquisa da MDER, a qual emitiu parecer favorável à sua realização.

No momento da inclusão no estudo, cada paciente era submetida, de forma aleatória, a tratamento anticonvulsivante com sulfato de magnésio ou com fenitoína.

As drogas que constituiam os dois esquemas terapêuticos foram distribuídas em caixas numeradas aleatoriamente, na proporção de um para um. Nas caixas contendo o sulfato de magnésio foram colocadas cinco ampolas de $10 \mathrm{~mL}$ a $50 \%$ contendo, cada uma, $5 \mathrm{~g}$ desta substância, além de cinco seringas de $10 \mathrm{~mL}$ que foram utilizadas para a administração das doses de ataque e de manutenção.

Nas caixas contendo o esquema da fenitoína foram colocadas oito ampolas de $5 \mathrm{~mL}$ contendo, cada uma, $250 \mathrm{mg}$ desta substância, além de uma seringa de $20 \mathrm{~mL}$ e quatro de $5 \mathrm{~mL}$, que foram utilizadas para a administração das doses de ataque e de manutenção.

Em todas as caixas foram colocadas folhas de papel que apresentavam descritos de forma clara os esquemas contidos em cada uma. Além disso, foram acrescidos a cada caixa pequena quantidade de areia e pedaços de madeira, a fim de que todas apresentassem aproximadamente o mesmo peso. Todas as caixas apresentavam o mesmo tamanho, a mesma forma e a mesma cor.

Até o momento da inclusão das pacientes no estudo, não se conhecia o conteúdo das caixas. À medida que cada paciente era admitida, uma caixa era aberta, aleatoriamente, e o esque- 
ma nela contido era administrado à paciente.

O sulfato de magnésio foi utilizado em dose de ataque de $5 \mathrm{~g}$ administrados por via endovenosa, seguida por doses de manutenção de $1 \mathrm{~g}$ por hora, em infusão venosa contínua. Para a administração da dose de ataque, $10 \mathrm{~mL}$ de sulfato de magnésio a $50 \%$ (5 g) eram diluídos em $100 \mathrm{~mL}$ de solução glicosada a $5 \%$ e infundidos a uma velocidade de 130 gotas por minuto. Na dose de manutenção, $10 \mathrm{~mL}$ de sulfato de magnésio a $50 \%$ (5 g) eram diluídos em $500 \mathrm{~mL}$ de solução glicosada a $5 \%$ e infundidos a uma velocidade de 28 gotas por minuto. Procurou-se não exceder a dose de $20 \mathrm{~g}$ em 24 horas.

A fenitoína foi utilizada na dose de ataque de $1.000 \mathrm{mg}$ diluídos em $100 \mathrm{~mL}$ de solução glicosada a $5 \%$ e administrada a uma velocidade de 130 gotas por minuto. A dose de manutenção consistia de $200 \mathrm{mg}$ da substância administrada por via endovenosa, a intervalos de 6 horas. Procurou-se não exceder a dose de 1.800 mg em 24 horas.

Nos casos de recidiva das convulsões era feita administração de diazepam na dose de 10 mg por via endovenosa.

Todas as pacientes incluídas no estudo foram avaliadas de acordo com os seguintes parâmetros: recidiva de convulsões após a instituição da terapia anticonvulsivante e existência de intercorrências clínicas durante a internação.

Em relação aos recém-nascidos foram avaliados os seguintes parâmetros: índice Apgar no primeiro e quinto minuto de vida e existência de intercorrências clínicas neonatais.

Para a análise estatística dos resultados foram utilizados os testes: diferenças das médias; com a finalidade de comparar os dois grupos em relação às características clínicas maternas, e teste para diferenças de proporções, com a finalidade de comparar os dois grupos em relação a ocorrência de intercorrências clínicas maternas e dos recém-nascidos e indice de Apgar. O teste $\mathrm{H}$ de Kruskal-Wallis foi empregado com a finalidade de comparar os dois grupos em relação à recidiva de convulsões.

Em todos os testes fixou-se em 0,05 ou $5 \%$ $(\mathrm{p} \leq 0,05)$ o nivel de rejeição à hipótese de nulidade.

\section{Resultados}

Nas tabelas a seguir encontram-se expostos os resultados obtidos para cada grupo de estudo.

A Tabela 1 apresenta os dados referentes às características clinicas das 77 pacientes que compõem os dois grupos. Nas pacientes tratadas com fenitoína, os valores médios para idade, número de gestações, número de crises convulsivas ocorridas antes da internação e número de partos normais são superiores aos verificados para os mesmos parâmetros no grupo tratado com sulfato de magnésio. A análise estatística não revelou, porém, diferença significante entre os grupos, no que se refere a estes dados $(\mathrm{p}>0,05)$.

Tabela 1 - Características clínicas das pacientes em cada um dos grupos de estudo.

\begin{tabular}{lrcrcc}
\hline Características clínicas & \multicolumn{2}{c}{ MgSO $_{4}$} & \multicolumn{2}{c}{ Fenitoína } \\
& Média & Desvio-padrão & Média & Desvio-padrão \\
\hline Idade (anos) & 18,7 & 4,1 & 20.4 & 5,9 & NS \\
Paridade & 1,3 & 0,7 & 1,6 & 0,9 & NS \\
Idade gestacional (semanas) & 36,6 & 3,1 & 36,1 & 3,7 & NS \\
Pressão arterial sistólica no momento da internação $(\mathrm{mmHg})$ & 163,4 & 22,7 & 156,1 & 13,3 & $p<0,05$ \\
Pressão arterial diastólica no momento da internação $(\mathrm{mmHg})$ & 110,2 & 17,8 & 109,2 & 11,5 & $\mathrm{NS}$ \\
Número de crises convulsivas ocorridas antes da internação & 2,2 & 1,4 & 2,3 & 1,6 & $\mathrm{NS}$ \\
Número de cesarianas & 0,8 & 0,4 & 0,8 & 0,4 & $\mathrm{NS}$ \\
Número de partos normais & 0,2 & 0,4 & 0,2 & 0,4 & $\mathrm{NS}$ \\
Pré-natal (n' de consultas) & 3,5 & 2,7 & 2,6 & 2,7 & $\mathrm{NS}$ \\
\hline
\end{tabular}

NS= não-significante

Em relação aos valores médios para idade gestacional, pressão arterial diastólica no mo- mento da internação, número de cesarianas e número de consultas ao pré-natal, estes foram 
superiores nas pacientes tratadas com sulfato de magnésio. A análise estatística também não indicou diferença significante entre os grupos no que se refere a estes dados $(p>0,05)$.

A média de pressão arterial sistólica no momento da internação, no grupo tratado com sulfato de magnésio foi $163,4 \mathrm{mmHg}$, ao passo que no grupo tratado com fenitoina este valor foi $156,1 \mathrm{mmHg}$. A diferença entre os grupos, em relação a este parâmetro, foi estatisticamente significante $(\mathrm{p}<0,05)$.

$\mathrm{Na}$ Tabela 2 encontram-se dispostos os dados relativos ao número de crises convulsivas ocorridas nas pacientes dos dois grupos, após a instituição do tratamento anticonvulsivante. No grupo tratado com fenitoína, $36,1 \%$ das pacientes apresentaram recidiva das convulsões, ao passo que no grupo tratado com sulfato de magnésio, somente $19,5 \%$ das mulheres tiveram novas crises. Quando se considerou a não-ocorrência de convulsão após a instituição do tratamento, $80,5 \%$ das pacientes tratadas com sulfato de magnésio não apresentaram crises convulsivas, ao passo que no grupo que recebeu fenitoína, 63,9\% das pacientes não apresentaram novas convulsões. Em relação a estes dados, a análise estatística revelou que a diferença entre os grupos é significante $(\mathrm{p}<0,05)$.

Tabela 2 - Distribuição das pacientes segundo o número de crises convulsivas ocorridas após instituição do tratamento anticonvulsivante.

\begin{tabular}{lrrrrl}
\hline Crises convulsivas & \multicolumn{2}{c}{$\mathrm{MgSO}_{4}$} & \multicolumn{2}{c}{ Fenitoína } & \\
& $\mathbf{n}$ & \multicolumn{1}{c}{$\%$} & \multicolumn{1}{c}{$\mathbf{n}$} & \multicolumn{1}{c}{$\%$} & \\
\hline Nenhuma & 33 & 80,5 & 23 & 63,9 & $\mathrm{p}<0,05$ \\
1 & 5 & 12,2 & 4 & 11,1 & $\mathrm{NS}$ \\
2 & 2 & 4,9 & 3 & 8,3 & $\mathrm{NS}$ \\
3 & 1 & 2,4 & 5 & 13,9 & $\mathrm{p}<0,05$ \\
4 & - & - & 1 & 2,8 & $\mathrm{NS}$ \\
Total & 41 & 100,0 & 36 & 100,0 & \\
\hline
\end{tabular}

NS = não-significante

A Tabela 3 apresenta os dados referentes à prevalência de intercorrências clínicas entre as pacientes dos dois grupos, durante a internação. Insuficiência renal e sindrome HELLP ocorreram nos dois grupos, tendo a insuficiência renal apresentado prevalência maior no grupo tratado com sulfato de magnésio, ao passo que edema pulmonar e coma ocorreram apenas nas pacientes tratadas com fenitoína. A análise estatística revelou que a diferença entre os grupos, em relação a estes dados, não é significante $(p>0,05)$.
No tocante à hemorragia pós-parto, observou-se que, nas pacientes tratadas com sulfato de magnésio, a prevalência foi de $14,7 \%$, ao passo que $2,7 \%$ das pacientes tratadas com fenitoina apresentaram tal intercorrência. A análise estatística revelou que esta diferença é significante $(p<0,05)$.

A Tabela 4 mostra os dados referentes ao

Tabela 3 - Distribuição das pacientes segundo a prevalência de intercorrências clínicas durantea internação.

\begin{tabular}{lcccrl}
\hline Intercorrências clínicas & \multicolumn{2}{c}{ MgSO $_{4}$} & \multicolumn{2}{c}{ Fenitoína } & \\
& $\mathbf{n}$ & $\%$ & $\mathbf{n}$ & $\%$ & \\
\hline Nenhuma & 31 & 75,6 & 30 & 83,2 & NS \\
Hemorragia & 6 & 14,7 & 1 & 2,8 & $p<0,05$ \\
Insuficiência renal & 3 & 7,3 & 1 & 2,8 & NS \\
Síndrome HELLP & 1 & 2,4 & 1 & 2,8 & NS \\
Edema pulmonar & - & - & 1 & 2,8 & NS \\
Coma & - & - & 2 & 5,6 & NS \\
Total & 41 & 100,0 & 36 & 100,0 & \\
\hline
\end{tabular}

NS = não-significante

índice de Apgar dos recém-nascidos dos dois grupos, no primeiro e quinto minuto de vida. No grupo tratado com fenitoína, $29,6 \%$ e $14,8 \%$ dos recém-nascidos apresentaram índice de Apgar menor que sete no primeiro e quinto minuto de vida respectivamente. Entre as pacientes que receberam sulfato de magnésio, $38,5 \%$ e $5,1 \%$ dos recém-nascidos apresentaram indice de Apgar menor que sete no primeiro e quinto minuto de vida respectivamente. A análise estatística revelou diferença significante entre os grupos quando foram considerados os valores relativos ao índice de Apgar menor que sete no quinto minuto de vida $(\mathrm{p}<0,05)$.

$\mathrm{Na}$ Tabela 5 encontram-se dispostos os

Tabela 4 - Distribuição dos recém-nascidos segundo o índice de Apgar no $1^{\circ} \mathrm{e} 5^{\circ}$ minutos de vida.

\begin{tabular}{lrrrrr}
\hline Índice de Apgar & \multicolumn{2}{c}{ MgSO $_{4}$} & \multicolumn{2}{c}{ Fenitoína } & \\
& $\mathbf{n}$ & \multicolumn{1}{c}{$\%$} & $\mathbf{n}$ & $\%$ & \\
\hline $1^{\circ}$ minuto $<7$ & 15 & 38,5 & 8 & 29,6 & NS \\
$\geq 7$ & 23 & 61,5 & 19 & 70,4 & NS \\
Total & 38 & 100,0 & 27 & 100,0 & \\
$5^{\circ}$ minuto $<7$ & 2 & 5,1 & 4 & 14,8 & p $<0,05$ \\
$\geq 7$ & 36 & 94,9 & 23 & 85,2 & NS \\
Total & 38 & 100,0 & 27 & 100,0 & \\
\hline
\end{tabular}

NS = não-significante

dados relativos à prevalência de intercorrências 
clínicas no período neonatal, nos recém-nascidos das pacientes que compõem os dois grupos de estudo. Cianose, hipoglicemia, hipotonia e infecção ocorreram com maior freqüência entre os recém-nascidos do grupo tratado com sulfato de magnésio. No grupo tratado com fenitoína não foram observados casos de icterícia, pneumonia e hipocalcemia, que ocorreram apenas nos recém-nascidos das mães tratadas com sulfato de magnésio. A prevalência de desconforto respiratório foi maior nos recém-nascidos do grupo tratado com sulfato de magnésio que naqueles do grupo tratado com fenitoína $(17,0 \%$ versus $11,8 \%)$. A análise estatística dos resultados não indicou diferença significante entre os grupos, em relação a estes parâmetros $(p>0,05)$.

Tabela 5 - Distribuição dos recém-nascidos, segundo a existência de intercorrências clínicas neonatais em cada um dos grupo de estudo.

\begin{tabular}{lrrrrr}
\hline Intercorrências clínicas & \multicolumn{2}{c}{ MgSO $_{4}$} & \multicolumn{2}{c}{ Fenitoína } & \\
& $\mathbf{n}$ & $\%$ & $\mathbf{n}$ & $\%$ & \\
\hline Nenhuma & 25 & 47,2 & 23 & 67,6 & NS \\
Desconforto respiratório & 9 & 17,0 & 4 & 11,8 & NS \\
Cianose & 6 & 11,4 & 1 & 2,9 & NS \\
Hipoglicemia & 3 & 5,6 & 2 & 5,9 & NS \\
Hipotonia & 3 & 5,6 & 2 & 5,9 & NS \\
Infecção & 3 & 5,6 & 2 & 5,9 & NS \\
Icterícia & 2 & 3,8 & - & - & NS \\
Pneumonia & 1 & 1,9 & - & - & NS \\
Hipocalcemia & 1 & 1,9 & - & - & NS \\
Total & $53^{*}$ & 100,0 & 34 & 100,0 & \\
\hline
\end{tabular}

*Alguns recém-nascidos apresentaram associação de intercorrências clínicas NS = não-significante

\section{Discussão}

Quando comparamos o efeito do sulfato de magnésio e da fenitoína sobre a recorrência de convulsões, observou-se diferença significativa, já que no grupo tratado com esta última, 13 pacientes apresentaram recidiva das crises $(36,1 \%)$, ao passo que no grupo tratado com sulfato de magnésio apenas oito mulheres apresentaram novas convulsões $(19,5 \%)$. Além disso, no grupo tratado com sulfato de magnésio, cinco das oito pacientes $(62,5 \%)$ apresentaram apenas uma convulsão, ao passo que nove das 13 pacientes $(69,2 \%)$ do grupo da fenitoína apresentaram entre duas e cinco crises convulsivas após a instituição da terapêutica.
Dados da literatura sugerem que o baixo desempenho anticonvulsivante da fenitoína em pacientes com eclâmpsia pode se dever ao fato de que as doses desta substância habitualmente empregadas não permitem a obtenção de níveis terapêuticos na circulação materna. Além disso, apesar da hipoalbuminemia em geral observada em pacientes com pré-eclâmpsia e eclâmpsia poder permitir uma maior fração de fenitoina livre, um maior volume de distribuição associado a clearance hepático e placentário aumentados desta substância observados na gravidez podem ser fatores que determinam a necessidade de doses maiores de fenitoína ${ }^{13}$.

No presente estudo, as doses de fenitoína utilizadas excederam, em muito, as doses relatadas em outros estudos ${ }^{9,12}$. Apesar disso, ainda se observou maior número de recidiva de convulsões no grupo tratado com esta substância. É provável, portanto, que a menor eficácia da fenitoína possa estar realmente relacionada às alterações no volume de distribuição e no clearance da substância relatado para mulheres com pré-eclâmpsia e eclâmpsia.

No tocante ao sulfato de magnésio, estudos recentes têm demonstrado sua superioridade em relação à fenitoína no controle e prevenção da recidiva de convulsões em mulheres com pré-eclâmpsia e eclâmpsia ${ }^{9,14}$.

Embora o mecanismo de ação do sulfato de magnésio permaneça desconhecido, sua superioridade como anticonvulsivante em relação a outras substâncias (diazepam, fenitoína) sugere que as convulsões apresentadas por mulheres com eclâmpsia podem ser diferentes, do ponto de vista fisiopatológico, da epilepsia ou outros tipos de convulsão ${ }^{15}$. O sulfato de magnésio pode atuar por meio de bloqueio neuronal dos canais de cálcio, reduzindo a isquemia cerebral. Além disso, esta substância pode induzir um aumento na produção e liberação de prostaciclina, inibir a agregação plaquetária e proteger o endotélio de danos mediados por radicais livres ${ }^{15,16}$.

Neste trabalho, das 8 pacientes que apresentaram recidiva das convulsões no grupo do sulfato de magnésio, apenas três tiveram mais de uma crise. A dose empregada correspondeu a uma infusão inicial de $5 \mathrm{~g}$, seguida de infusão venosa contínua de $1 \mathrm{~g}$ por hora. Existem relatos de que o sulfato de magnésio pode ser utilizado em infusão de $2 \mathrm{~g}$ por hora, sem a ocorrência de alterações compativeis com intoxicação pelo íon magnésio $^{2}$. É provável, portanto, que a ocorrência de recidiva de convulsões neste grupo esteja associada à dose utilizada da substância.

Em relação aos efeitos do sulfato de magnésio e da fenitoína sobre o organismo materno, 
observou-se que $83,8 \%$ das pacientes no grupo tratado com fenitoína e $75,6 \%$ daquelas que utilizaram sulfato de magnésio não apresentaram qualquer intercorrência. No entanto, $14,7 \%$ das mulheres do grupo de sulfato de magnésio apresentaram hemorragia pós-parto, ao passo que apenas $2,7 \%$ das pacientes tratadas com fenitoína apresentaram tal intercorrência.

Os dados acerca dos efeitos do sulfato de magnésio sobre o organismo materno são escassos, sendo descrita a ocorrência de ondas de calor, cefaléia, secura na boca, vertigens e letargia, quando da infusão da dose de ataque ${ }^{17}$. Sabese, no entanto, que o sulfato de magnésio suprime a contratilidade de fibras miometriais isoladas in vitro, atuando provavelmente de forma competitiva com o cálcio, diminuindo as concentrações livres deste íon no meio intracelular que são necessárias para a interação actino-miosina no processo de contratilidade da musculatura lisa ${ }^{18}$. É provável que, atuando desta maneira, o sulfato de magnésio tenha levado à ocorrência de atonia uterina, o que pode ter determinado a maior prevalência de hemorragia pós-parto nas pacientes tratadas com esta substância. Embora seja descrito que a fenitoína possa interferir no metabolismo da vitamina $\mathrm{K}$ e induzir a alterações dos mecanismos de coagulação vitamina Kdependentes ${ }^{19}$, não se observaram, no presente estudo, taxas elevadas de hemorragia pós-parto, no grupo de pacientes que utilizaram este fármaco.

Quando se compararam os efeitos do sulfato de magnésio e da fenitoína sobre os resultados perinatais, observou-se que, no grupo tratado com fenitoina, um número maior $(14,8 \%)$ dos recém-nascidos apresentou índice de Apgar menor que sete no quinto minuto de vida, em comparação ao grupo que utilizou sulfato de magnésio. Estes dados estão de acordo com os descritos pelo The Eclampsia Trial Collaborative Group. É provável que o maior índice de recidiva de convulsões no grupo tratado com fenitoina tenha contribuído para uma maior prevalência de sofrimento fetal, o que pode ter determinado este maior percentual de recém-nascidos deprimidos.

Em relação às intercorrências neonatais observamos que $47,2 \%$ dos recém-nascidos de mães que usaram sulfato de magnésio e 67,6\% daqueles do grupo tratado com fenitoína não apresentaram intercorrências. No entanto, 17,0\% dos recém-nascidos do grupo do sulfato de magnésio apresentaram desconforto respiratório, ao passo que $11,8 \%$ daqueles do grupo da fenitoína manifestaram tal intercorrência. Da mesma forma, a ocorrência de cianose foi maior nos recémnascidos do grupo do sulfato de magnésio que no grupo da fenitoína, embora a diferença não seja estatisticamente significante. Sabe-se que o sulfato de magnésio atravessa rapidamente a placenta e seus níveis no feto e recém-nascido crescem proporcionalmente aos niveis séricos maternos ${ }^{20}$. Agindo de forma similar à descrita para a musculatura uterina, o sulfato de magnésio pode ter determinado certo grau da supressão da musculatura intercostal e diafragmática destes neonatos, determinando, assim, esta maior prevalência de dificuldade respiratória e cianose.

Observa-se, portanto, que o sulfato de magnésio apresenta-se como droga mais eficaz que a fenitoína no controle e prevenção da recidiva de convulsões em pacientes com eclâmpsia. Sua utilização esteve associada a uma maior prevalência de hemorragia pós-parto, dificuldade respiratória no neonato e cianose, embora estas intercorrências não tenham contribuído para a ocorrência de óbitos maternos e neonatais. Os dados ora apresentados confirmam estudos prévios que sugerem que o sulfato de magnésio deve ser a droga de escolha para o tratamento de eclâmpsia.

\section{SUMMARY}

Purpose: to compare the efficiency between magnesium sulfate and phenytoin in the control of convulsions in patients with eclampsia and to evaluate the effects of magnesium sulfate and phenytoin on the maternal and perinatal prognosis in patients with eclampsia.

Methods: this is a prospective, randomized and controlled study in which the results obtained with the use of anticonvulsive treatment in 77 women with eclampsia, treated with either magnesium sulfate or phenytoin, were analyzed comparatively. The drugs which were used in both therapeutic schemes were distributed in a one to one ratio, in randomly numbered boxes which presented similar characteristics. When a patient was admitted, a box was opened and its contents were given to the patient.

Results: in the group whose patients were treated with magnesium sulfate, $19.5 \%$ had recurrent convulsions while in the group whose patients used phenytoin, $36.1 \%$ had new crises $(p<0,05)$. The patients who were treated with magnesium sulfate showed a greater prevalence of postpartum hemorrhage $(14,7 \%)$ than those to whom phenytoin was administered $(2.7 \%)(p<0.05)$. In relation to the newborns, $17.0 \%$ of the group from mothers treated with magnesium sulfate presented respiratory distress as opposed to the group of newborns from mothers treated with phenytoin $(11.8 \%),(p>0,05)$. 
Conclusion: magnesium sulfate is shown to be more efficient than phenytoin in the control and the prevention of convulsions in patients with eclampsia. However, its utilization showed a higher prevalence of postpartum hemorrhage and respiratory distress. Phenytoin should be used in cases where the use of magnesium sulfate is contraindicated.

KEY WORDS: Magnesium sulfate. Phenytoin. Eclampsia. Hypertension. Puerperium. Respiratory distress syndrome.

\section{Referências}

1. Neme B. Doença hipertensiva específica da gestação: pré-eclâmpsia e eclâmpsia. In: Rezende J, editor. Obstetrícia. $6^{a}$ ed. Rio de Janeiro: Guanabara Koogan; 1991. p.488-522.

2. Sibai BM. Hipertensão na gravidez. Clin Obstet Ginecol Am Norte 1992; 4:651-67.

3. Bergström S, Povey G, Songane F, Ching C. Seasonal incidence of eclampsia, its relationship to meteorological data in Mozambique. J Perinat Med 1992; 20:153-8.

4. Geographic variation in the incidence of hypertension in pregnancy. World Health Organization International Collaborative Study of Hypertensive Disorders of Pregnancy. Am J Obstet Gynecol 1988; 158:80-3.

5. Douglas KK, Redman CWG. Eclampsia in the United Kingdom. BMJ 1994; 309:1395-400.

6. Cecatti JG, Albuquerque RM, Hardy E, Faúndes A. Mortalidade materna em Recife. Causas de óbitos maternos. Rev Bras Ginecol Obstet 1998; 20:7-11.

7. Valadares-Neto JD, Martins MPSS, Valadares SM, Costa-Filho NF. Mortalidade materna na Maternidade Dona "Evangelina Rosa" (TeresinaPiauí). Rev Bras Ginecol Obstet 1995; 17:97784.
8. Duley L. Maternal mortality associated with hypertensive disorders of pregnancy in Africa, Asia, Latin America and the Caribbean. Br J Obstet Gynaecol 1992; 99:547-53.

9. Which anticonvulsant for women with eclampsia? Evidence from the Collaborative Eclampsia Trial. Lancet 1995; 345:1455-63.

10. Chesley LC. Hypertensive disorders in pregnancy. New York: Appleton-Century-Crofts; 1978. History; p.17-34.

11. Slater RM, Wilcox FL, Smith WD, et al. Phenytoin infusion in severe preeclampsia. Lancet 1987; $1: 1417-21$

12. Dommisse J. Phenytoin sodium and magnesiun sulfate in the management of eclampsia. Br J Obstet Gynaecol 1990; 97:104-9.

13. Sankar R, Licht EA. Magnesiun sulfate versus phenytoin for the prevention of eclampsia. N Engl J Med 1995; 333:1638-9.

14. Lucas MJ, Leveno K, Cunningham FG. A comparison of magnesium sulfate with phenytoin for the prevention of eclampsia. N Engl J Med 1995; 333:201-5.

15. Higgins JR, Brennecke SP. Preeclampsia and eclampsia: magnesium salts for all? Med J Aust 1998; 168:151-2.

16. Lipton SA, Rosenberg PA. Excitatory amino acids as a final common pathway for neurologic disorders. N Engl J Med 1994; 330:613-22.

17. Creasy RK. Preterm labor and delivery. In: Creasy RK, Resnik R, editors. Maternal-Fetal Medicine. $2^{\text {nd }}$ ed. Philadelphia: W.B.Saunders; 1989. p.477504.

18. Huszar G. Physiology of the myometrium. In: Creasy RK, Resnik R, editors. Maternal-Fetal Medicine. $2^{\text {nd }}$ ed. Philadelphia: W. B. Saunders; 1989. p.141-8.

19. Watson JD, Spellacy WN. Neonatal effects of maternal treatment with the anticonvulsant drug diphenylhydantoin. Obstet Gynecol 1971; 37:881-5.

20. Cruikshank DP, Pitkin RM, Reynolds WA, Williams GA, Hargis GK. Effects of magnesium sulfate treatment on perinatal calcium metabolism. I. Maternal and fetal responses. Am J Obstet Gynecol 1979; 134:243-9. 